\title{
Product Module Partition Methodology Based on Function-Principle-Structure
}

\author{
Jing CHEN \\ Guilin University of Technology \\ School of Mechanical Control Engineering \\ Guilin 541004, China \\ jingc812@163.com
}

\author{
Wen-bo WANG \\ Guilin University of Technology \\ School of Mechanical Control Engineering \\ Guilin 541004, China \\ 251661096@qq.com
}

\begin{abstract}
In this paper we analyzed the product design process model based on function-principle-structure. Regarding the existing products as an object for module partition, we researched the related principles and quantitative method based on the function, principle, structure between the two parts. In the research of structural-relation, the structure related principles and quantitative method based on the assembly relation were proposed. We constructed the correlation sub-matrix for function, principle and structure, weighted the correlation sub-matrix and generated comprehensive correlation matrix; According to the comprehensive correlation matrix, we calculated the maximum correlation between parts. With average correlation for threshold, we proposed product module partition methods and steps based on clustering analysis, and realized product module. Finally, we use the case of speed reducer to verify the effectiveness of the module partition method.
\end{abstract}

Keywords - function-principle-structure; assembly relation; clustering analysis; module partition

\section{INTRODUCTION}

Modular technology can effectively support the rapid design of products, which is one of the key technologies to achieve mass customization. The basic idea is dividing the product into a series of modules. Inside the module, the relationship is closely linked but the relationship between the module is loose. Through the different combinations of modules, we can get different function of the product [1]. And reasonable module division is the prerequisite and foundation of the mapping of the custom product domain, which will directly affect the function, performance and cost of the product. The division of the module is the key to the modular design of the product. At present, the common module division method includes complex network technology [1], fuzzy clustering method [2], heuristic methods for identifying modules for product architectures [3].

The modularization method based on the function-principle-structure model conforms to the designers' habits of thinking. Therefore, it is necessary to consider the function, principle and structure of the product in order to get a more reasonable division result. Through a series of components of the assembly relationship, products achieve the function of the product. Therefore, the module interface depends on a series of components of the assembly relationship to achieve the function of the product. So, when the module is divided, it is necessary to consider the assembly information between the modules. At present, the research on the method of module division mainly uses the qualitative judgment on the structural correlation between components; however consideration of the impact of the number of assembly information between components of the relevant structure is not abundant. In this paper, considering the assembly relationship and the number of components, the components of the product are divided into modules and the product module division method based on function-principle-structure is studied.

\section{CORRELATION PRINCIPLE BETWEEN COMPONENT AND} QUANTIFICATION METHOD BASED ON FUNCTION - PRINCIPLE STRUCTURE

\section{A. Relevance Principle and Quantitative Method Base on \\ Function}

The principle of function-related principle is that gathering of the components which achieve a certain or some of the requirements for a module to meet the requirements of the product requirements. The principle can guarantee the functional independence of the module. Functional correlation and quantization value are shown in Table I.

TABLE I. THE QUANTITATIVE VALUE AND FUNCTION CORRELATION

\begin{tabular}{|c|c|}
\hline Functional relevance & $\begin{array}{c}\text { Quantitation } \\
\text { values }\end{array}$ \\
\hline There are interdependencies between components & 1.0 \\
\hline $\begin{array}{c}\text { Parts have a strong collaborative relationship } \\
\text { between }\end{array}$ & $0.5 \sim 0.9$ \\
\hline There is a weak relationship between parts & $0.1 \sim 0.4$ \\
\hline $\begin{array}{l}\text { Parts are independent of each other without } \\
\text { functional relationship }\end{array}$ & 0 \\
\hline
\end{tabular}

The functional correlation quantization is a range value, and the specific value is determined by the degree of correlation. According to the function-related principle and the quantification method, the function correlation between the parts is evaluated and quantified by the designer, and the functional sub-correlation matrix between the components is constructed by the quantization result $R_{f}=\left(r_{i j}^{f}\right)_{n \times n}$, its matrix elements $\quad r_{i j}^{f}(i=1,2, \ldots, n ; j=1,2, \ldots, n)$ functional relevance quantization value between $\mathrm{x}_{\mathrm{i}}$ and $\mathrm{x}_{\mathrm{j}}$. 


\section{B. Principle of relevance and quantitative method based on principle}

Product development process usually includes a variety of scientific techniques and methods, therefore, its modular process should be fully considered principle which is used in the principle and follow the related principle. In the modular process, the principle is that having the components of the same or similar principles should form a module. For example, in the product development and design phase, the module components should contain similar principles of application and technical staff should develop intensely. In the product processing and manufacturing stage, the module components should have the same processing and manufacturing technology. The correlation value of the principle correlation is shown in Table II

TABLE II. THE QUANTITATIVE VALUE OF PRINCIPLE CORRELATION

\begin{tabular}{cc}
\hline Principle relevance & Quantitation values \\
\hline Components use the same principle & 1.0 \\
Parts with similar principles & $0.5 \sim 0.9$ \\
Parts of the principle of using a certain & $0.1 \sim 0.4$ \\
connection & 0 \\
\hline
\end{tabular}

According to the related principle and the method of quantification, the designer evaluates and quantifies the principle relevance between components, and uses its quantization results to construct the principle sub-correlation matrix between parts $R_{p}=\left(r_{i j}^{p}\right)_{n \times n}$. Its matrix elements

$r_{i j}^{p}(i=1,2, \ldots, n ; j=1,2, \ldots, n)$ is principle relevance quantization value of $\mathrm{x}_{\mathrm{i}}$ and $\mathrm{x}_{\mathrm{j}}, \mathrm{n}$ is the amount of the components.

\section{Structure - based Relevance Principle and Quantification Method}

The assembly relationship information refers to the contact information which is due to the assembly between the parts of the product, including the relationship, the assembly geometry constraint, the connection relation and the motion relation information [4]. The common assembly relationship of mechanical products can be divided into connection relationship, movement relationship, position relationship. The specific description product assembly relationship can see [5].

\section{1) Structural Relativity Principle and Quantification} Method Based on Assembly Relation

The structure-related principle is that the structure of the module has some correlation, and the overall structure of the module is relatively independent. This is the most basic principle of module partitioning, which ensures the structural integrity and independence of each module.

Through the interface between components, which is a series of assembly relations, product achieves the function of the product. The function of the assembly relationship is essentially achieved by component structure as a carrier. Therefore, the assembly relationship between parts reflects how the structure implements the function, and reflects the structural relevance between the components. Different assembly relationship types and accuracy requirements reflect the structural correlation between components, and depending on the type and accuracy requirements of the assembly relationship the structural relational quantization values of the assembly relationship are shown in Table III

TABLE III. THE QUANTITATIVE VALUE OF STRUCTURE CORRELATION BASED ON ASSEMBLY RELATION

\begin{tabular}{cc}
\hline Assembly relationship & $\begin{array}{c}\text { Quantitative } \\
\text { values }\end{array}$ \\
\hline $\begin{array}{c}\text { Tightly connected, hard to disassemble. Such as } \\
\text { welding, interference connection } \\
\text { With tolerance requirements. Such as key } \\
\text { connection, gear drive and so on }\end{array}$ & 1.0 \\
$\begin{array}{c}\text { With no tolerance requirements. Such as thread } \\
\text { connections }\end{array}$ & $0.5 \sim 0.9$ \\
Not connected & $0.1 \sim 0.4$ \\
\hline
\end{tabular}

In addition, there may be a variety of assembly relationships between components, and the larger the number of assembly relationships is, the greater the structural correlation between components is. So both the assembly relationship and its number reflect the structural correlation between components. In this paper, we use the assembly relationship between components and their quantity to measure the structural correlation. The quantitative correlation between the parts is the sum of the quantitative values of the structural correlation between the components. The larger the value is, the greater structural correlation between components is.

\section{2) Constructing structural sub - correlation matrices}

According to the structure-related principles and quantification methods of the components, the structural correlations between the components are evaluated and quantified by the designer, and the initial structure correlation matrix between the parts is constructed by using the quantization results $\left(\mathrm{R}_{s}\right)_{0}$. Its matrix elements $\left(r_{i j}^{s}\right)_{0}(\mathrm{i}=1,2, \ldots, \mathrm{n}$; $\mathrm{j}=1,2, \ldots, \mathrm{n} ; \mathrm{i} \neq \mathrm{j}$ ) is Initial structure correlation quantization value between $\mathrm{x}_{\mathrm{i}}$ and $\mathrm{x}_{\mathrm{j}}$, and mapping matrix element values to interval $[0,1]$. The process is essentially the smallest - the most normalized mapping process, and its mathematical expression is

$$
\begin{gathered}
r_{i j}^{s}=\frac{\left(r_{i j}^{s}\right)_{0}-\left(r_{i j}^{s}\right)_{\min }}{\left(r_{i j}^{s}\right)_{\max }-\left(r_{i j}^{s}\right)_{\min }}\left(\left(r_{i j}^{s}\right)_{\max } \neq\left(r_{i j}^{s}\right)_{\min }\right) \\
r_{i j}^{s}(i=1,2, \ldots, n ; j=1,2, \ldots, n, i \neq j) \quad \text { is the quantization of the }
\end{gathered}
$$
structural correlation between the $\mathrm{xi}$ and $\mathrm{xj}, \mathrm{n}$ is the amount of the parts ; $\left(r_{i j}^{s}\right) \max ,\left(r_{i j}^{s}\right)$ min are the maximum and minimum matrix element values of the matrix (Rs) 0 respectively.

$r_{i j}^{s}$ is matrix elements, and defining the structural relevance of the parts themselves are 1. Structure between the sub-correlation matrix is $R_{s}=\left(r_{i j}^{s}\right)_{n \times n}$. 
D. Comprehensive correlation matrix based on function principle - structure

The correlation distance between the parts $\mathrm{x}_{\mathrm{i}}$ and $\mathrm{x}_{\mathrm{j}}$ is calculated by the weighted sum of the quantization of the function, principle and structure. The formula is

$$
\begin{array}{r}
d_{i j}= \begin{cases}\omega_{f} r_{i j}^{f}+\omega_{p} r_{i j}^{p}+\omega_{s} r_{i j}^{s} & (i \neq j) \\
1 & (i=j)\end{cases} \\
\left(0 \leq d_{i j} \leq 1 ; i, j \in\{1,2, \ldots, n\}\right)
\end{array}
$$

$\omega_{f}, \omega_{p}, \omega_{s}$ are the sub-factors of the function, principle, structure respectively. $\omega_{f}+\omega_{p}+\omega_{s}=1 ; r_{i j}^{f}, r_{i j}^{p}, r_{i j}^{s}$ are the quantization value of the function, principle, and structure of the parts $\mathrm{x}_{\mathrm{i}}$ and $\mathrm{x}_{\mathrm{j}} . \mathrm{N}$ is the number of parts. The correlation distance $\mathrm{d}_{\mathrm{ij}}$ between the parts $\mathrm{x}_{\mathrm{i}}$ and $\mathrm{x}_{\mathrm{j}}$ represent the matrix elements. The synthetic correlation matrix of the components is constructed

$$
\mathrm{D}=\left[\begin{array}{cccc}
d_{11} & d_{12} & \cdots & d_{1 n} \\
d_{21} & d_{22} & \cdots & d_{2 n} \\
\vdots & \vdots & & \vdots \\
d_{n 1} & d_{n 2} & \cdots & d_{n n}
\end{array}\right]
$$

$\mathrm{D}$ is a symmetric matrix, reflecting the comprehensive relationship between the parts based on function, principle, and structure.

\section{Product Module Division Based on Cluster ANALYSIS}

\section{A. Comprehensive correlation matrix transformation}

The synthetic correlation matrix D reflects the fuzzy similarity relation between the samples, but it only has the reflexivity and symmetry, but not the transitivity. Therefore, the comprehensive correlation matrix $\mathrm{D}$ is transformed into the fuzzy equivalent matrix $D^{*}[6]$. The transformation method is starting from the comprehensive correlation matrix D, followed by seeking its fuzzy square, the mathematical expression is

$$
\left\{\begin{array}{l}
D \rightarrow D^{2} \rightarrow D^{4} \rightarrow \cdots \\
D^{2}=D \circ D=\vee\left(d_{i j} \wedge d_{j i}\right)
\end{array}\right.
$$

$\circ$ is Zade operator; $\vee$ is logic plus, which is taking large on; $\wedge$ is the logic minus, which is taking small one.

When the $D^{t} \circ D^{t}=D^{t}$ first appears. Indicating that $D^{t}$ is already transitive, and $D^{t}$ is the required transitive closure, which is the fuzzy equivalent matrix $\mathrm{D}^{*}$. The composite correlation matrix D is transformed into a fuzzy equivalence matrix by the above method $D^{*}=\left(d_{i j}^{*}\right)_{n \times n}$.

\section{B. Method and Step of Product Module Partitioning Based on Cluster Analysis}

According to the fuzzy equivalence matrix $\mathrm{D}^{*}$, the product components are analyzed by the calculation of the maximum correlation between the parts of the method, product module division of the specific steps are:

\section{1) Determine the threshold for clustering analysis.}

There are some uncertainties in the manual setting threshold. By calculating the value of the correlation distance between the components as the threshold of the cluster analysis, the average correlation is calculated by:

$$
K=\frac{\sum_{i=1}^{n} \sum_{\substack{j=1 \\ i \neq j}}^{n} d_{i j}^{*}}{n(n-1)}
$$

2) Calculate the pair of components with the greatest degree of correlation and mix them into a module.

By calculating the largest element in the fuzzy equivalent matrix $\mathrm{D}^{*}$, the parts $\mathrm{x}_{\mathrm{p}}, \mathrm{x}_{\mathrm{q}}$ with the largest degree of correlation are obtained:

$$
T_{(p, q)}=\max \left\{d_{i j}^{*}(i=1,2, \ldots, n ; j=1,2, \ldots, n ; i \neq j)\right\}
$$

Since the correlation between the components $\mathrm{x}_{\mathrm{p}}, \mathrm{x}_{\mathrm{q}}$ is the largest, $\mathrm{x}_{\mathrm{p}}, \mathrm{x}_{\mathrm{q}}$ is a module and is denoted as $\mathrm{M}(\mathrm{p}, \mathrm{q})$.

The method of dealing with the original matrix is:

a) Get rid of the $p, q$ rows and the $p, q$ columns in the matrix $D^{*}$

b) Calculate the correlation distance between module $M$ $(p, q)$ and the rest of the components Its formula is:

$$
d_{i M}^{*}=\max \left\{d_{i p}^{*}, d_{i q}^{*}(i=1,2, \ldots, n ; i \neq p \neq q)\right\}
$$

c) The module $M(p, q)$ is added to the matrix and the correlation distance between the module $M(p, q)$ and the remaining components is taken as the element of the module $M(p, q)$ in the matrix.

The order of the new matrix is n-1 after processing.

3) Similarly, the use of Step2 method can calculate the largest degree of correlation of a pair of parts or modules, and they are clustered into a module. Using Step 2 on the original matrix processing methods gets $n-2$ order matrix.

4) Treating the average correlation degree $K$ as the threshold, the clustering algorithm process is ended until the maximum correlation degree in the matrix is less than the average degree of correlation $K$, according to Step 2 and Step 3 cycle operation. The final matrix for the results of cluster analysis which is the results of the module division can be got.

\section{EXAMPLE ANALYSIS}

Taking the gearbox as an example illustrates the product module division method. The main components of the gearbox are shown in Fig. I. 

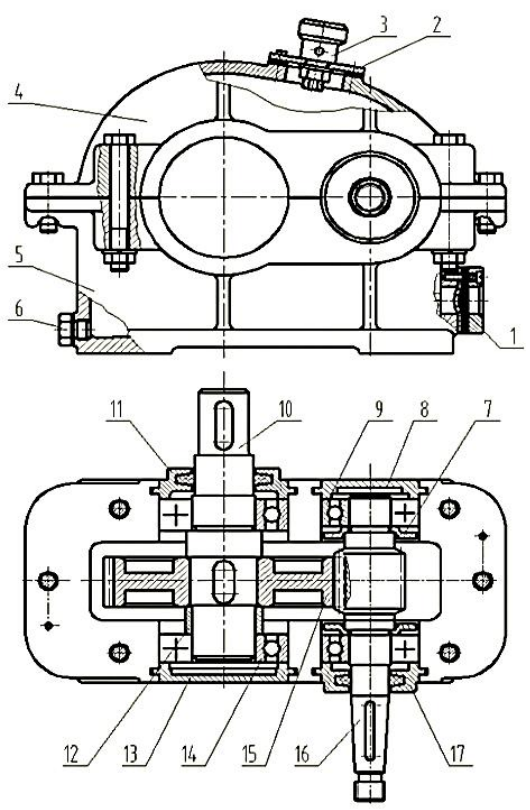

Fig. 1. Main parts of speed reducer

TABLE IV. MAIN PARTS NAME AND NUMBER OF SPEED REDUCER

\begin{tabular}{cccc}
\hline $\begin{array}{c}\text { Serial } \\
\text { number }\end{array}$ & name & $\begin{array}{c}\text { Serial } \\
\text { number }\end{array}$ & name \\
\hline 1 & Oil level indicator & 2 & As the hole cover \\
3 & Ventilator & 4 & Cover \\
5 & Base & 6 & Oil plug \\
7 & Retaining ring & 8 & High-speed bearing \\
stuffy cover
\end{tabular}

According to the function, principle, structure related principle and quantization method, the function, principle and structure sub-correlation matrix between parts are constructed. According to the analytic hierarchy process [7], the weight of function, principle and structure correlation is $0.38,0.38$; The use of equation (2) can calculate the correlation distance between components $d_{i j}$, and using formula (3) build the comprehensive correlation matrix $\mathrm{D}$, and then using equation (4) can get the fuzzy equivalent of the components between the components. The use of (5) calculate the average degree of correlation $\mathrm{K}=0.382$, then using the formula (6) calculate the largest degree of correlation of a pair of parts $\mathrm{T}(10,15)=0.924$ Parts 10 and 15 are clustered into one module. The maximum number of elements in the fuzzy equivalent matrix $\mathrm{D}^{*}$ is 0.380 , when maximum correlation is less than the average degree of correlation $\mathrm{K}$, then the clustering algorithm is terminated and the final clustering result is obtained. The gearbox module is divided. The cluster diagram is shown in Fig. II. According to the actual project, less relevant components is usually classified as accessories, so the parts 1, 2, 3, 6, 7 are an annex module.
Fig. III shows the results of the gearbox module. The module division result of the gearbox is: high speed shaft $(8,9,16,17)$, low speed shaft $(10,11,12,13,14,15)$, Supporting the box (4, $5)$, accessories $(1,2,3,6,7)$ four modules. The results of this module are similar to those based on engineering practice and experience.

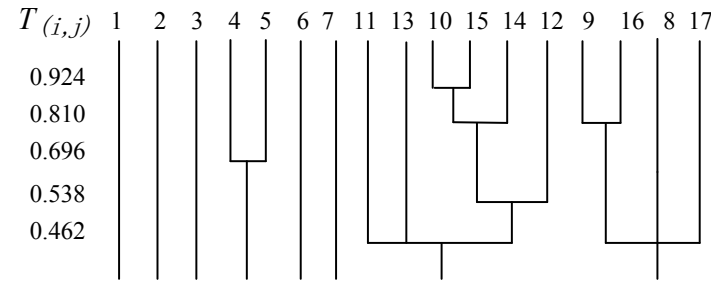

Fig. 2. The dynamic clustering diagram of speed reduce

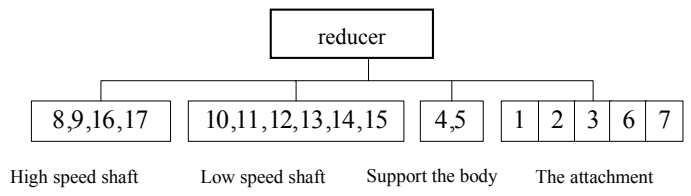

Fig. 3. The module partition result of speed reducer

\section{CONCLUSION}

In this paper, we study the correlation between the components based on function, principle and structure, and propose the structure-related principles and quantization methods of components based on assembly relations in structural correlation research. Function, principle, structure sub-correlation matrix is constructed. Finally, according to the comprehensive correlation matrix carrying out clustering analysis, the division of the product module is realized. The validity of the method is verified by the example of the division of the gearbox module. The division method can provide support for modular product design.

\section{REFERENCES}

[1] Yujun Lu, Chengyu Lei, Jianxin Gu, Guoning Qi, Modular design method of order product based on maximum fitness optimization algorithm. J. Computer integrated manufacturing system. vol. 19, 2013, pp. 909-917.

[2] Weiqiang Jia, Zhenyu Liu, Daxin Liu, Gong Xun, Jianrong Tan, Modular design method of complex product and its application based on Fuzzy Association J. Chinese Journal of Mechanical Engineering. vol. 51, 2015, pp. 130-142.

[3] ROBERT B S, KRISTIN L W, RICHARD H C, A heuristic method for indentifying modules for product architectures J. Design Studies. vol. 21 2000, pp. 5-31.

[4] Xiaoyi Wang, Huifen Wang, Youliang Zhang, Quanxian Wang, Reconfigurable assembly model for assembly planning J. Computer integrated manufacturing system. vol. 17, 2011, pp. 53-61.

[5] Chen Jing, Liu Li, Cheng Yang, Zhiwei Gong, Research on product model supporting scheme design and technology design J. machine design. vol. 28, 2011, pp. 1-6.

[6] Xianfu Cheng, Chen Cheng, Product module partition method based on design correlation matrix and extension clustering J. machine design. vol 29, 2012, pp. 5-9.

[7] Haijun Wang, Baoyuan Sun, Analysis of product modularization process based on Fuzzy Clustering J. Computer integrated manufacturing system. vol. 9, 2003, pp. 123-126. 